\title{
Stox1 as a novel transcriptional suppressor of Math1 during cerebellar granule neurogenesis and medulloblastoma formation
}

\author{
Chenlu Zhang ${ }^{1}$, Zhongzhong Ji ${ }^{1}$, Minglei Wang ${ }^{1}$, Weiwei Zhang ${ }^{1}$, Rong Yang ${ }^{2}$, Huanping $\mathrm{An}^{3}$, Ru Yang ${ }^{1}$, Daan van Abel ${ }^{4}$, \\ Marie van Dijk ${ }^{4}$, Xiaohang Yang ${ }^{3,5}$, Guangshuo Ou ${ }^{6}$, Helen He Zhu ${ }^{\star, 1}$ and Wei-Qiang Gao ${ }^{*, 1,2,7}$
}

Cerebellar granule neuronal progenitors (GNPs) are the precursors of cerebellar granule cells (CGCs) and are believed to be the cell of origin for medulloblastoma (MB), yet the molecular mechanisms governing GNP neurogenesis are poorly elucidated. Here, we demonstrate that storkhead box 1 (Stox1), a forkhead transcriptional factor, has a pivotal role in cerebellar granule neurogenesis and MB suppression. Expression of Stox1 is upregulated along with GNP differentiation and repressed by activation of sonic hedgehog (SHH) signaling. Stox1 exerts its neurogenic and oncosuppressing effect via direct transcriptional repression of Math1, a basic helix-loop-helix transcription activator essential for CGC genesis. This study illustrates a SHH-Stox1-Math1 regulatory axis in normal cerebellar development and MB formation.

Cell Death and Differentiation (2016) 23, 2042-2053; doi:10.1038/cdd.2016.85; published online 26 August 2016

Postnatal cerebellum is a supreme model to study neurogenesis owing to its simple and well-defined laminar structure. Cerebellar granule cells (CGCs) differentiated from granule neuronal progenitors (GNPs) are the most abundant neuronal cell type in the central nervous system. ${ }^{1}$ The renewal expansion and differentiation of GNPs are intricately regulated spatiotemporal processes. ${ }^{2-4}$ GNPs proliferate actively on the cerebellar surface after birth to generate the external granule layer (EGL). ${ }^{5} \mathrm{EGL}$ peaks at postnatal day 7 and persists until the 3rd week in mice. We demonstrated in our recent study that GNPs undergo mostly symmetric division during early postnatal EGL development, and switch gradually to asymmetric division to produce one GNP and one intermediate cell that sequentially exits cell cycle and differentiates. ${ }^{6}$ The differentiated CGCs extend the parallel and radial fibers, then migrate inward from EGL through the molecular layer (ML) and Purkinje cell layer $(\mathrm{PL})$ to form the internal granule layer (IGL). ${ }^{7}$

Aberrant proliferation and/or blocked differentiation of GNPs can lead to the development of MB, the most deadly brain cancer in children. Signaling pathways that act to control normal cerebellar neurogenesis are often deregulated in MB oncogenesis, yet the key molecules involved remain poorly identified. Based on the transcriptional profiling of human MB samples, MBs are classified into four molecular subtypes with distinct mRNA expression signatures associated with sonic hedgehog, WNT, Myc signalings or undefined genetic anomalies. $^{8-10} \mathrm{SHH}$, secreted by Purkinje cells, is a crucial cytokines in promoting GNP amplification and inhibiting differentiation through downstream genes, such as Gli1, Math1, Ccnd1 and MycN. ${ }^{11} \mathrm{SHH}$ signaling pathway stands out as the most attractive therapeutic target for MB, as it is the most prevalent molecular subtype of MB. Activation of $\mathrm{SHH}$ pathway by smothened (SMO) or Patched gene deletion led to MB development in mouse models. ${ }^{12,13}$ Although small molecule SHH pathway inhibitors have been shown to effectively suppress $M B$ in the mouse and human, drug resistance and aggressive disease remission inevitably occur, which are mainly caused by mutations in SMO. ${ }^{14,15}$ Therefore, novel targets are in urgent need for the treatment of MBs with $\mathrm{SHH}$ signature.

Math1, a basic helix-loop-helix (bHLH) transcription factor specifically expressed in the proliferating GNPs, is a commonly used cerebellar EGL marker. ${ }^{16,17}$ It is required for both the expansion and differentiation suppression of GNPs. In addition, Math1 expression is markedly increased in the $\mathrm{SHH}$-activated subtype of MB. ${ }^{12,18}$ It was reported that Math1 acts in the SHH MB development by modulating the expression of cell cycle progression genes $\mathrm{Ccnd} 2$ and $\mathrm{SHH}$ downstream signaling genes Gli2. ${ }^{19,20}$ Bone morphogenic proteins (BMPs) and Wnt signaling have been demonstrated to posttranslationally regulate the Math1 protein abundance through proteasome-mediated degradation in MB or colon cancer. ${ }^{18,21}$

\footnotetext{
${ }^{1}$ State Key Laboratory of Oncogenes and Related Genes, Renji-Med X Clinical Stem Cell Research Center, Ren Ji Hospital, School of Medicine, Shanghai Jiao Tong University, Shanghai 200127, China; ${ }^{2}$ School of Biomedical Engineering \& Med-X Research Institute, Shanghai Jiao Tong University, Shanghai 200030, China; ${ }^{3} \mathrm{College}$ of Life Sciences, Zhejiang University, Hangzhou 310058, China; ${ }^{4}$ Department of Clinical Chemistry, VU University Medical Center, de Boelelaan 1117, Amsterdam 1081 HV, the Netherlands.; ${ }^{5}$ Institute of Genetics, School of Medicine, Zhejiang University, Hangzhou 310058, China; ${ }^{6}$ Tsinghua-Peking Center for Life Sciences, School of Life Sciences, Tsinghua University, Beijing 100084, China and ${ }^{7}$ Collarative Innovation Center of Systems Biomedicine, Shanghai, China

${ }^{*}$ Corresponding author: HH Zhu or W-Q Gao, State Key Laboratory of Oncogenes and Related Genes, Renji-Med X Clinical Stem Cell Research Center, Ren Ji Hospital, School of Medicine, Shanghai Jiao Tong University, 160 Pujian Rd., Shanghai 200127, China. Tel: +86 21 68383917; Fax: +86 21 68383916;

E-mail: zhuhecrane@shsmu.edu.cn or gao.weiqiang@sjtu.edu.cn

Abbreviations: GNPs, granule neuronal progenitors; CGCs, cerebellar granule cells; MB, medulloblastoma; SHH, sonic hedgehog; EGL, external granule layer; ML, molecular layer; PL, Purkinje layer; IGL, internal granule layer; SMO, smothened; bHLH, basic helix-loop-helix; BMPs, bone morphogenic proteins; Stox1, Storkhead box 1; ChIP, chromatin immunoprecipitation

Received 06.2.16; revised 02.7.16; accepted 14.7.16; Edited by M Freeman; published online 26 August 2016
} 
Still, much is unknown about the key component in the transcriptional activation or repression of Math1 gene in morphogenic or oncogenic cerebellum development.

Storkhead box 1 (Stox1), a winged helix transcription factor closely related to the forkhead protein family, was originally found to be associated with the preeclampsia disorder characterized by gestational hypertension and proteinuria. ${ }^{22}$ It was recently reported that Stox 1 is abundantly expressed in the brain and upregulated in the Alzheimer's disease. ${ }^{23}$ HAM1, the ortholog of Stox1 in the $c$. elegans, has been shown to control the neuroblast cell fate by regulating the spindle position and myosin polarization. ${ }^{24}$ Recent study suggested that the role of HAM1 or Stox1 in neuronal migration appeared to be conserved between $C$. elegans hermaphrodite specific neurons and Xenopus neural crest cells. ${ }^{25}$ However, the role of Stox 1 in mammalian neurogenesis is poorly defined. In this study, we explore the role of Stox1 in the cerebellum neurogenesis and MB development.

\section{Results}

Stox1 is expressed in granule neurons of EGL in postnatal developing cerebellum. We first determined the expression pattern of Stox 1 in the developing cerebellum.
Cerebella from postnatal day P0, P6, P14, P21 and P60 were harvested for immunoblotting. As shown in Figure 1a, the expression of Stox1 in mouse cerebellum increased at the first 2 weeks after birth, but declined later on. Owing to the lack of high-quality anti-Stox 1 antibody suitable for immunostaining of tissue section, we utilized quantitative RT-PCR and immunoblotting to evaluate the expression level of Stox1 in different compartments of cerebellum fractionated by percoll gradient sedimentation or flow cytometry sorting. Cerebella of day 6 were collected and digested with papain followed by $35 \% / 60 \%$ percoll gradient centrifugation. The GNPs from EGL were enriched at the interface between 35 and $60 \%$ percoll with a purity $>90 \%$, whereas the remaining cerebellar cells mainly from Purkinje's cells and interneurons stayed at the top of the gradient. ${ }^{26}$ As shown in Figure 1b, strong expression of Stox1 was found in EGL. EGL is comprised of proliferating GNPs and newly differentiated Dcx-expressing granule neurons. ${ }^{27}$ To further determine the expression pattern of Stox1 in EGL, we performed quantitative RT-PCR of flow cytometry sorted the Dcx-positive and -negative CGCs from Dcx-DsRed mice. Stox1 was more abundantly expressed in newly differentiated Dcx-positive granule neurons than Dcx-negative GNPs (Figure 1c). In addition, GNPs from Math1-GFP transgenic mouse were
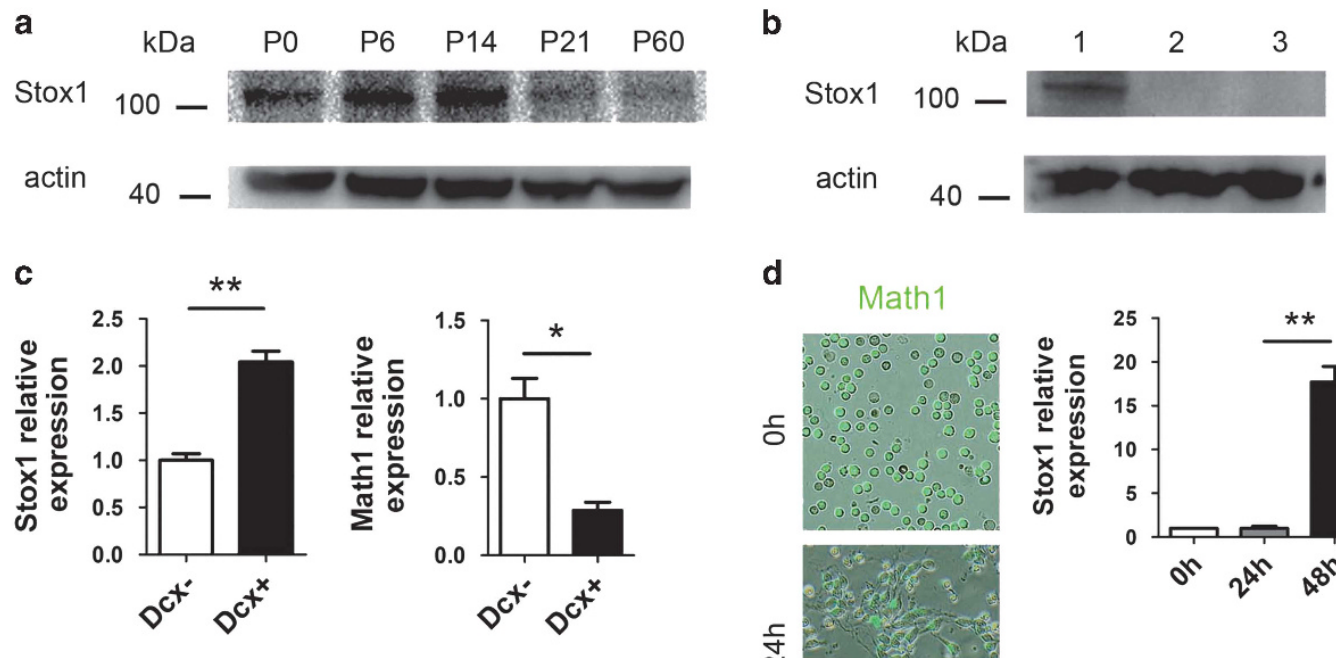

d
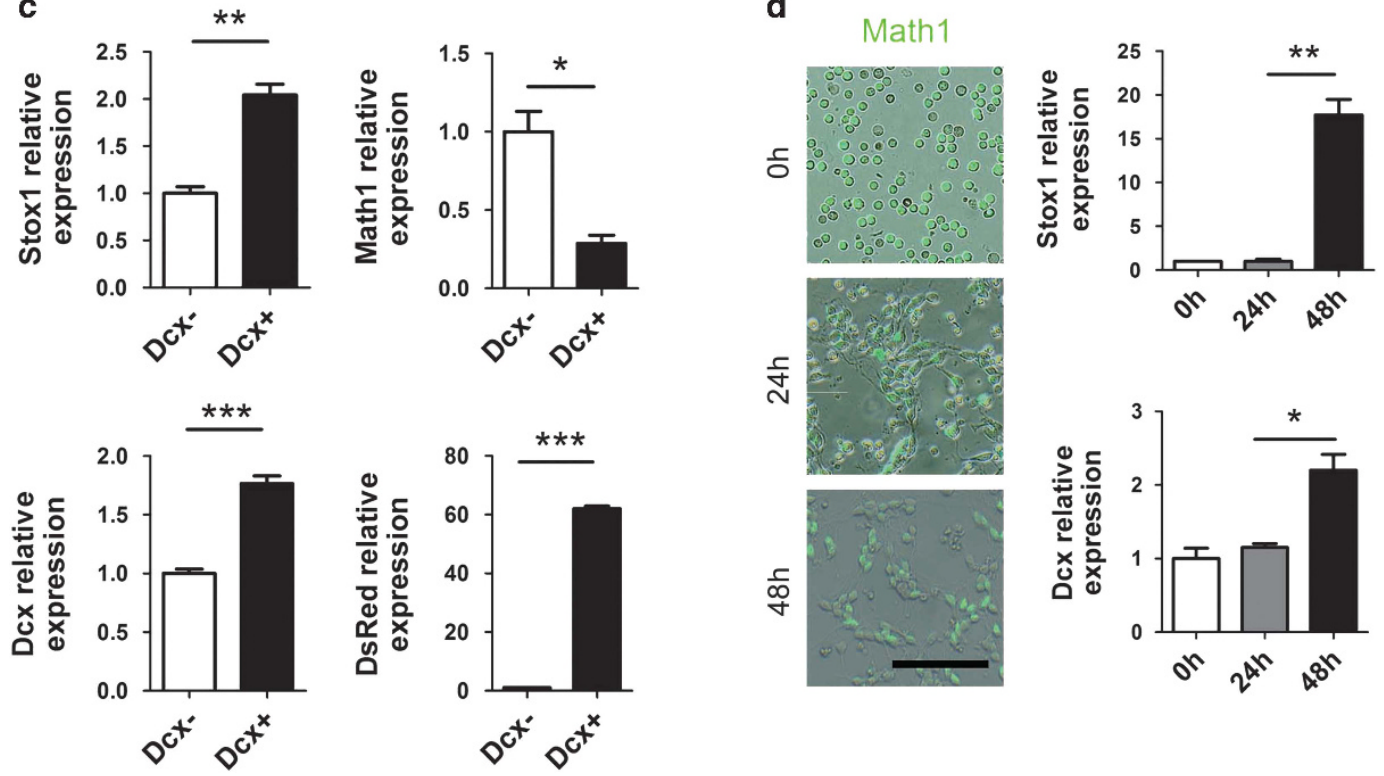

Figure 1 Stox 1 is expressed in granule neurons of EGL in the postnatal cerebellum. (a) Immunobloting of Stox1 in P0, P6, P14, P21 and adult mouse cerebellums shows that the expression level of Stox1 increases during the first 2 weeks after birth, but declines thereafter. (b) Immunobloting data show strong expression of Stox1 in EGL. Lane 1: GNPs, Lane 2: Purkinje cells and interneurons, Lane 3: spleen as negative control. (c) Dcx-positive and -negative granule cells from Dcx-DsRed mice were enriched using the flow cytometry sorting. Quantitative real-time PCR analysis shows significantly higher expression of Stox1, Dcx and DsRed and lower expression of Math1 in Dcx-positive granule cells compared with Dcx-negative GNPs. $(n=6)$ (d) GNPs of Math1-GFP mice were collected for in vitro differentiation. The mRNA expression of Stox1 and Dcx increases along the 2 day differentiation. Scale bars $=100 \mu \mathrm{m} .(n=6) .\left({ }^{* \star *} P<0.001,{ }^{* \star} P<0.01,{ }^{\star} P<0.05\right.$, data are shown as means \pm S.E.M. $)$ 
a

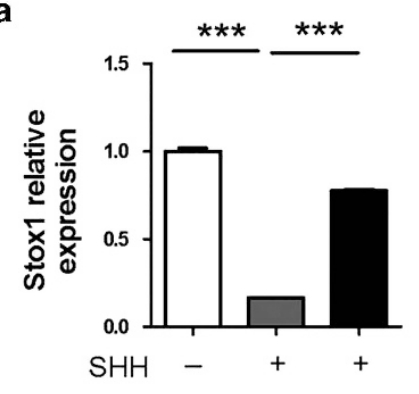

cyclopamine $\quad-\quad-\quad+$

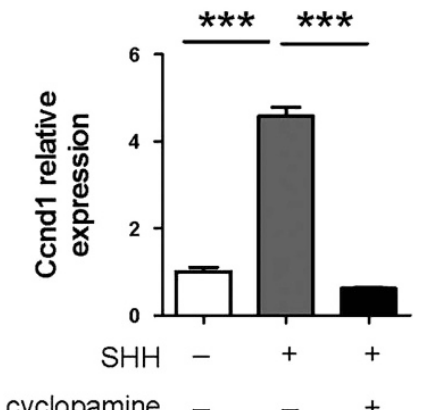

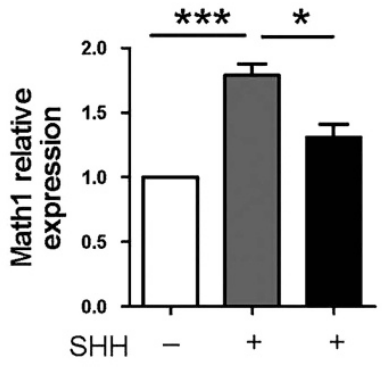

cyclopamine - $\quad+$

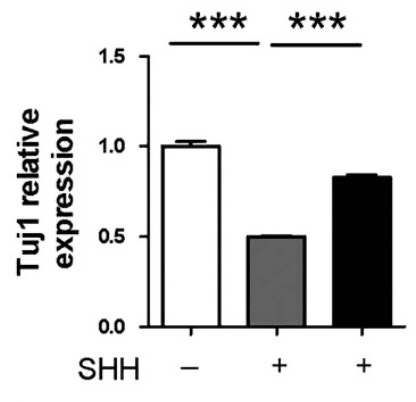

b

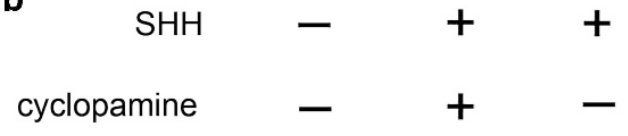

\section{$\mathrm{kDa}$}

Stox1

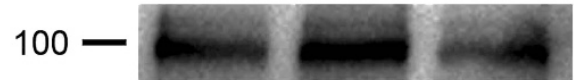

actin

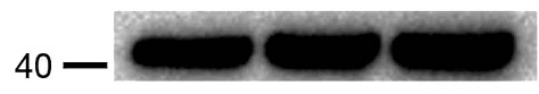

Figure 2 The expression of Stox1 is suppressed by SHH signaling. (a) SHH stimulation leads to repressed expression of Stox1 and Tuj1 but enhanced expression of Math1 and Ccnd1. Cyclopamine, a SHH signaling inhibitor, abrogates the impact of SHH treatment on the expression of Stox1, Math1, Ccnd1 and Tuj1. Dissociated culture of mouse GNPs is treated with $1 \mu \mathrm{g} / \mathrm{ml} \mathrm{SHH}$ alone or together with $5 \mu \mathrm{M}$ cyclopamine. After $48 \mathrm{~h}$ of treatment, mRNA was extracted for quantitative RT-PCR analysis. (b) Immunobloting data indicates that $\mathrm{SHH}$ stimulation upregulates the expression of Stox1 and downregulates the expression of Math1, whereas cyclopamine abrogates the impact of SHH treatment

enriched and subjected to in vitro differentiation for 2 days. Quantitative RT-PCR demonstrated an upregulation of Stox1 and Dcx along the differentiation of GNPs (Figure 1d). Taken together, Stox1 was strongly expressed in the EGL of the postnatal cerebellum, with the highest level detected in Dcxpositive inner EGL granule neurons.

The expression of Stox 1 is modulated by SHH signaling. It was shown previously that the $\mathrm{SHH}$ pathway has a crucial role in cerebellar granule neurogenesis. ${ }^{1}$ In the developing cerebellum, proliferation of GNPs and the expression of GNP marker Math1 are stimulated with $\mathrm{SHH}$ signaling. ${ }^{28} \mathrm{We}$ therefore tested if Stox1 was modulated by the $\mathrm{SHH}$ signal pathway. As shown in Figure 2a, treatment of dissociated cultures of GNPs with SHH led to enhanced expression of Math1 and Ccnd1, whereas SHH stimulation resulted in suppressed expression of differentiated granule neuron maker Tuj1. Interestingly, the expression of Stox1 was significantly repressed in SHH treated primary GNPs (Figures $2 \mathrm{a}$ and $\mathrm{b}$ ). Addition of a $\mathrm{SHH}$ signaling inhibitor cyclopamine compromised the impact of $\mathrm{SHH}$ treatment on the expression of Stox1, Math1, Ccnd1 and Tuj1 (Figures 2a and b), suggesting a $\mathrm{SHH}$ signaling specific effect on the modulation of Stox1 expression.

Stox1 promotes the differentiation of cerebellar granule progenitor cells. GNPs are located at EGL in the postnatal mouse cerebellum, which continue to proliferate and differentiate during the postnatal $2-3$ weeks. ${ }^{1}$ Math1 is specifically expressed in GNPs and frequently used as a GNP marker, ${ }^{29,30}$ whereas Dcx is found to be expressed specifically in the early differentiated GNPs. ${ }^{31-33}$ To analyze the biological function of Stox1 in postnatal cerebellar neurogenesis, we constructed a Stox1-expressing adenovirus that co-expressed tomato red fluorescent protein as a reporter. Math1-GFP and Dcx-DsRed reporter mice in which the Math1 or Dcx expressing granule neurons can be readily identified using respective fluorescent protein were introduced from the Jackson Laboratory. ${ }^{30,33}$ GNPs from day 6 Math1-GFP reporter mice were purified by percoll gradient sedimentation method, ${ }^{34}$ plated on poly-D-lysine coated plates, and then infected with Stox1-expressing adenovirus. Stox1 overexpression was validated by quantitative RT-PCR and immunobloting (Figure 3a). Infected GNPs were collected at $48 \mathrm{~h}$ and subjected to flow cytometric analysis of the Math1-GFP fluorescent intensity. As shown in Figure 3b, the Math1-GFP level was reduced in Stox1-overexpressing GNPs (Figure 3b). Consistently, quantitative PCR revealed a significant downregulation of Math1 mRNA level in Stox1 adenovirus-infected GNPs. In contrast, overexpression of Stox1 resulted in an upregulation of the differentiated CGC markers including Dcx, Tuj1, Neurod1 and NeuN (Figure 3c). In line with that, immunofluorescent staining showed enhanced generation of Dcx and Tuj1-positive cells from Stox1-overexpresing GNPs, suggesting a neurogenic effect of Stox1 (Figure 3d).

To further test the hypothesis that Stox1 functions as a positive regulator in GNP differentiation, we used a 
loss-of-function approach by constructing an adenovirus containing shRNA against Stox1 that co-expressed a GFP fluorescent reporter. GNPs from $D c x-d s R e d$ reporter mice were purified and infected with shStox1 adenovirus. Efficient Stox1 gene silencing was confirmed by quantitative PCR and immunobloting (Figure 4a). Flow cytometric analysis showed a significant reduced generation of Dcx-positive cells from GNPs upon Stox1 knockdown (Figure 4b). Differentiation from GNPs into Dcx and NeuN-positive cerebellar granule neurons was similarly repressed by Stox1 knockdown
(Figures 4c and d). Consistent with that, quantitative RTPCR exhibited an upregulation of Math1 and a downregulation of differentiated cerebellar granule neuron markers Dcx, Tuj1, NeuN, Neurod1, Zic1 and Zic2 from in vitro culture of GNPs infected with shStox1 adenovirus (Figure $4 \mathrm{e}$ ). The relative moderate effect of shStox1 compared with forced Stox1 expression on Math1 and Dcx-dsRed expression was likely due to redundant signaling molecules or transcriptional regulators besides Stox1 in promoting GNP differentiation. Altogether, those data identified Stox1 as a proneurogenic

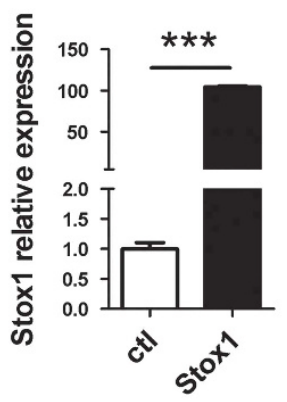

b

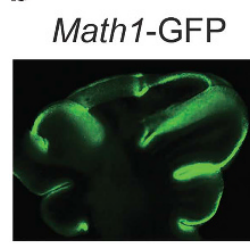

GNPs enrichment by percoll
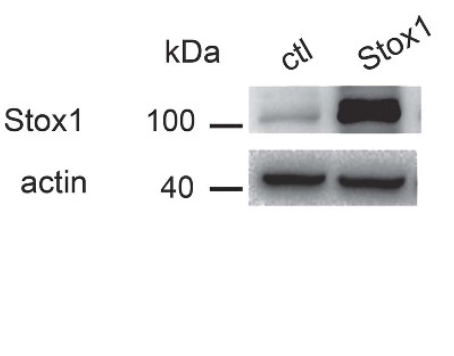

\section{C}
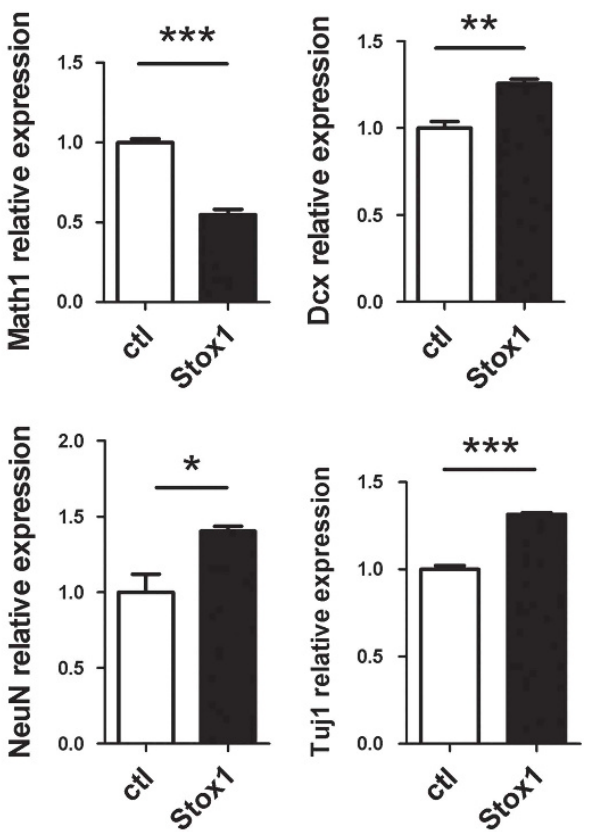

Stox1 expressing adenovius infection

\section{GFP} expression analysis

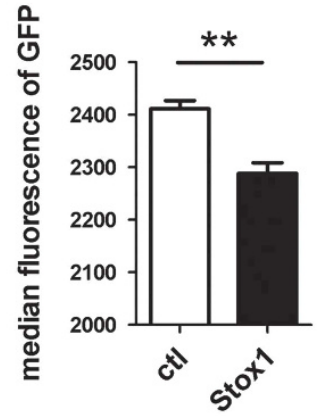

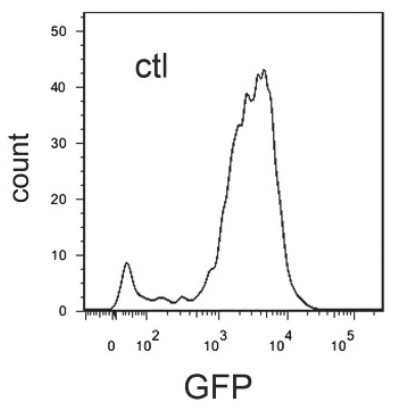

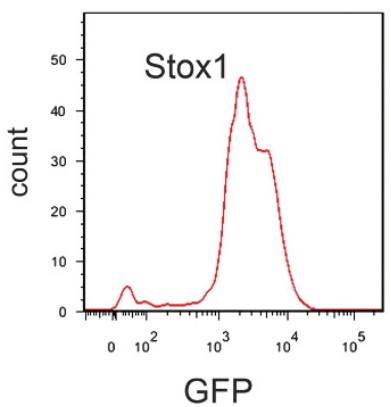

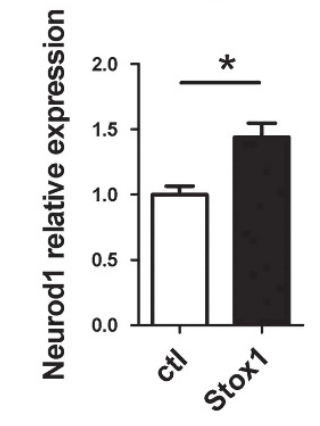
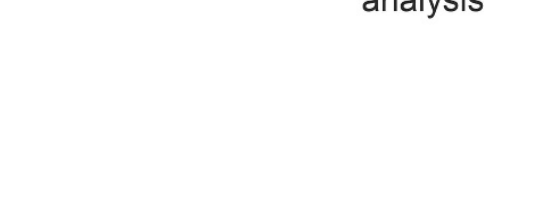
d

Tuj1
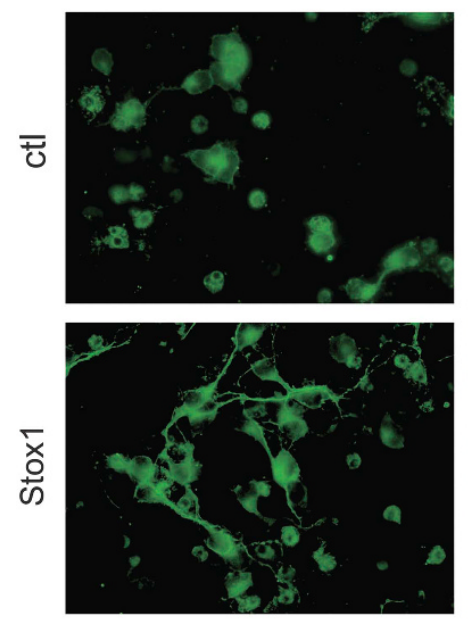

e
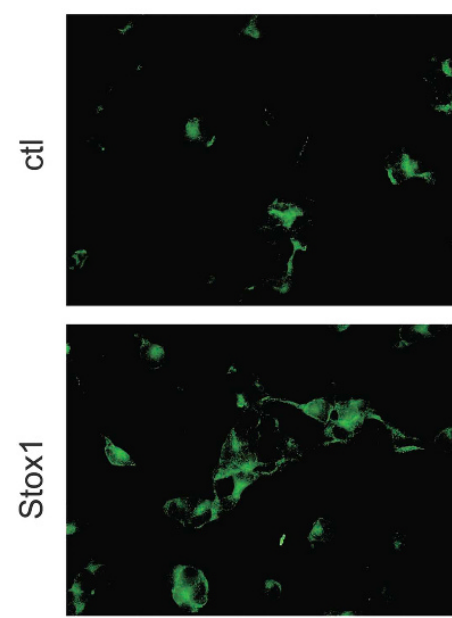

tdTomato
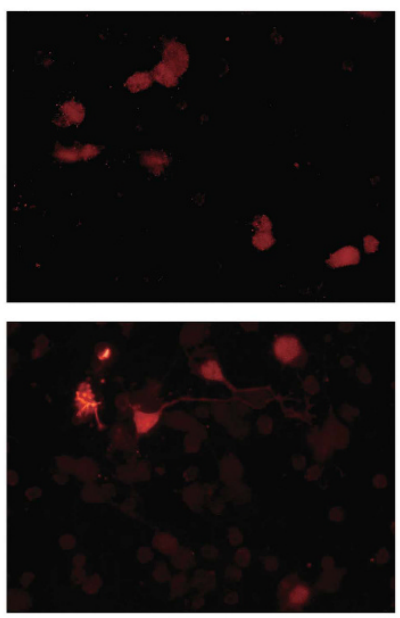

tdTomato
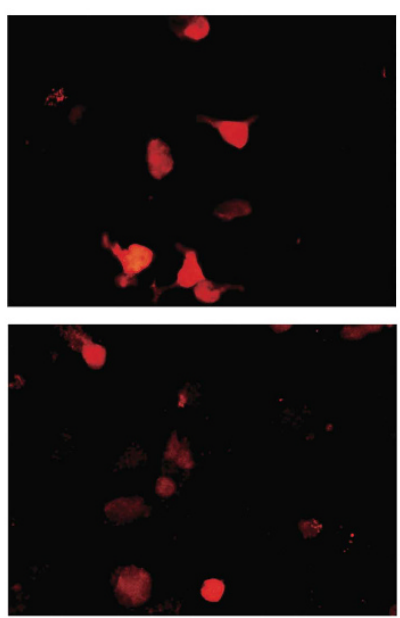

DAPI
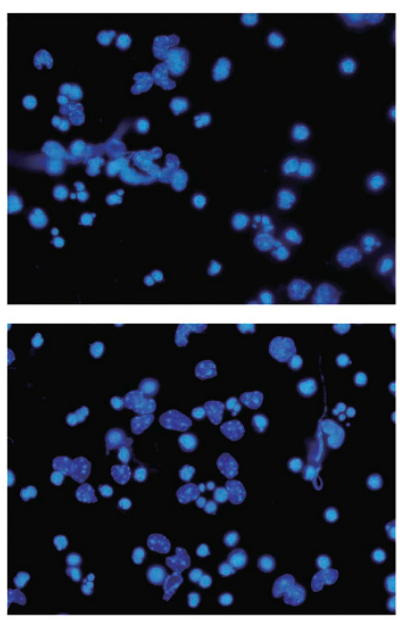

DAPI
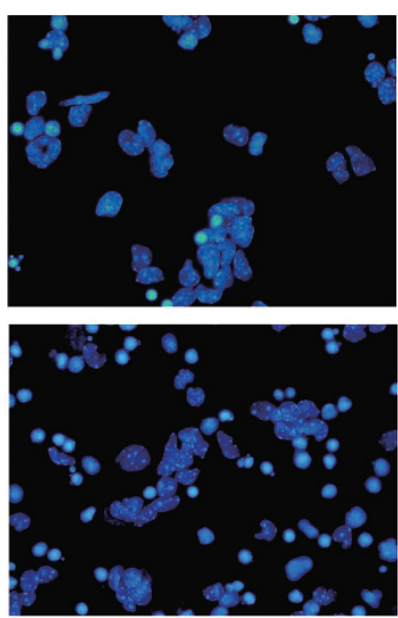

Merge
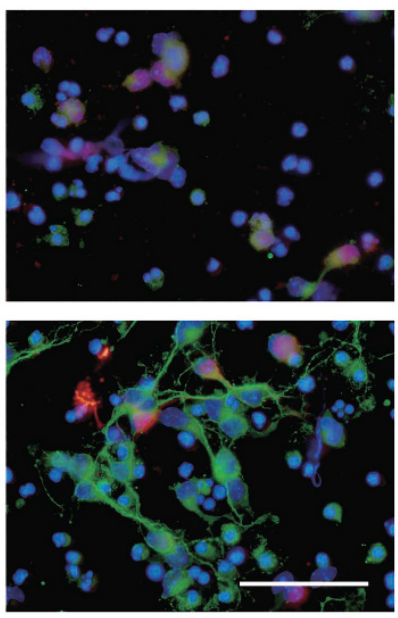

Merge
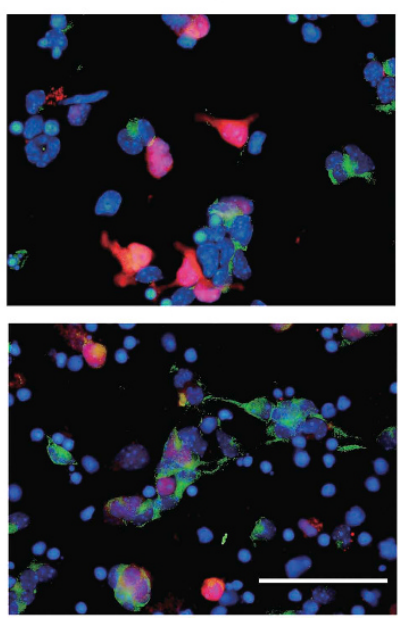

Figure 3 Continued

factor in the cerebellum. Considering cell cycle exit and differentiation of GNPs are usually paralleled biological processes, we explored whether Stox1 affected the proliferation pattern of GNPs. As shown in Figure 4f, Stox1 knockdown resulted in a strikingly increased mitotic activity in GNPs, determined by elevated percentage of cells incorporated with EdU. Consistently, the expression of Ccnd1, a key factor in promoting cell cycle progression, was significantly elevated upon Stox1 knockdown, whereas the expression of cell cycle inhibitors p18, p21 and p27 were downregulated in shStox1 adenovirus-infected GNPs (Figure $4 \mathrm{~g}$ ).

Stox1 serves as a transcriptional suppressor of Math1. Stox1 is a member of the forkhead transcriptional factor family whose canonical target DNA-binding sequence is RYMAAYA. ${ }^{35,36}$ Using this sequence, we did in silico analysis of key genes involved in GNP proliferation and differentiation in searching for potential Stox1 downstream targets. Interestingly, we found multiple potential Stox1-binding sites in the cis-regulatory region of Math1 gene. It was previously reported that two conserved fragments (referred to as enhancer $A$ and enhancer $B$ ) within the $1.7 \mathrm{~kb}$ fragment at $3.4 \mathrm{~kb}$ 3' of the Math1-coding region, sufficient for driving a lacZ reporter to recapitulate the endogenous Math1 expression in mice, was the key cis-regulatory element of Math1. ${ }^{37}$ To test if Stox1 functioned as a trans-acting factor of Math1, we took advantage of a reporter plasmid containing GFP coding sequence driven by the $1.7 \mathrm{~kb}$ Math 1 cis-regulatory fragment comprised of enhancer A, enhancer B and the sequence in-between (termed as fragment C) (Figure 5a). Co-transfection of the Math1-GFP reporter plasmid and Stox1-expressing adenovirus led to repressed expression of GFP, whereas infection of shStox1 adenovirus resulted in increased GFP expression in GNPs (Figure 5a). These data were confirmed by luciferase assays showing that Stox1 negatively regulated the luciferase reporter driven by the $1.7 \mathrm{~kb}$ Math1 cis-regulatory element (Figure 5b). Collectively, these data suggested that Stox 1 acted as a tran-regulatory 
factor of Math1. To further delineate functional regulatory regions within the $1.7 \mathrm{~kb}$ Math 1 enhancer regulated by Stox1, we constructed luciferase reporter plasmids containing enhancer $A$, enhancer $B$ or fragment $C$, respectively. As shown in Figure 5b, infection of Stox1 adenovirus downregulated the luciferase reporter activity driven by enhancer $A$ and enhancer B, but not fragment C. Consistently, Stox1 interference by shStox 1 enhanced the luciferase reporter a

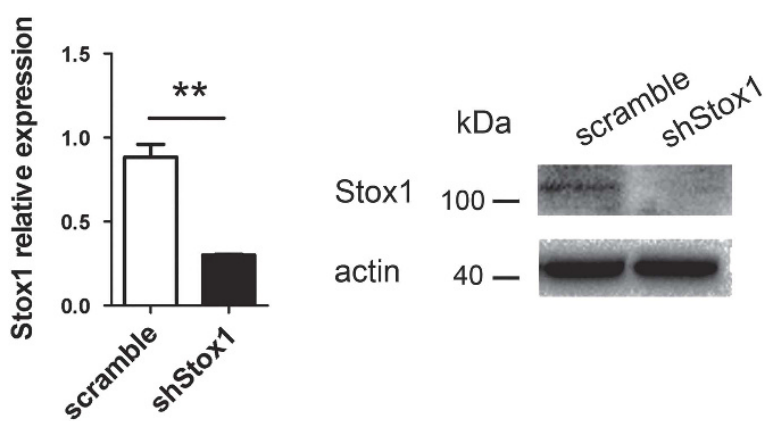

b
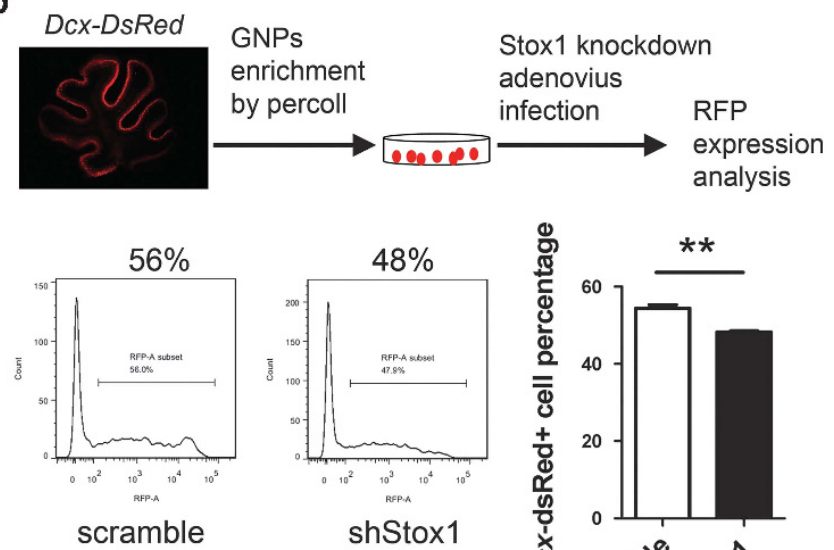

C
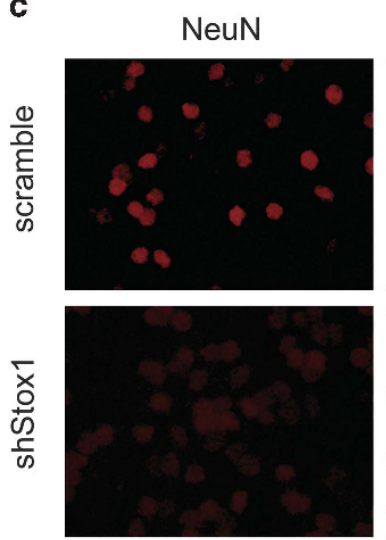

d
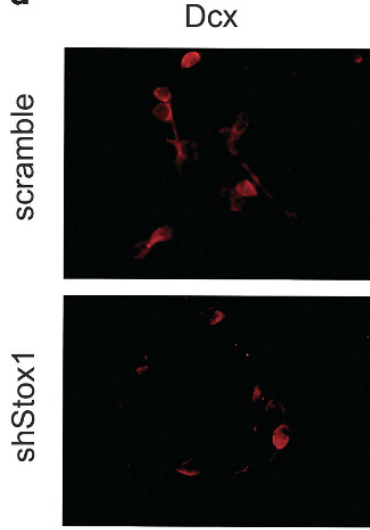

GFP
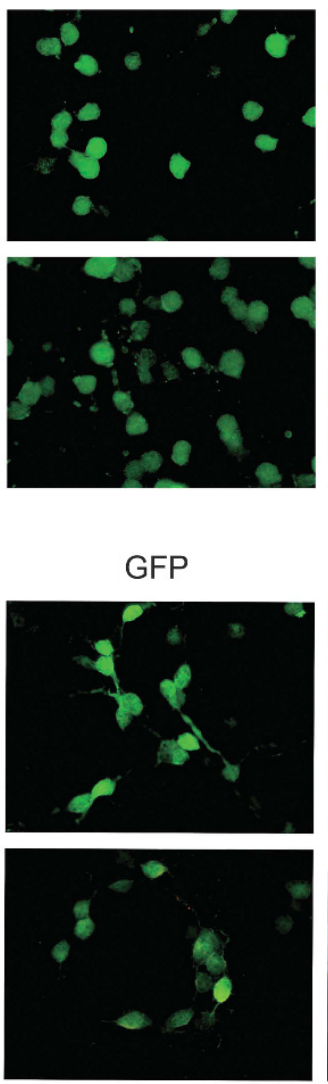

DAPI
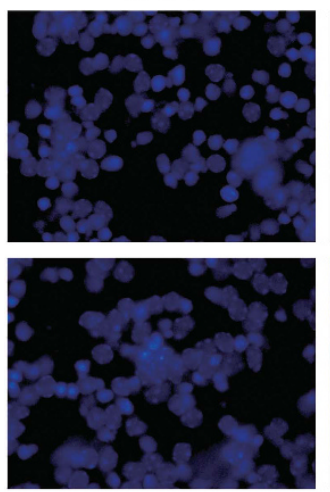

DAPI
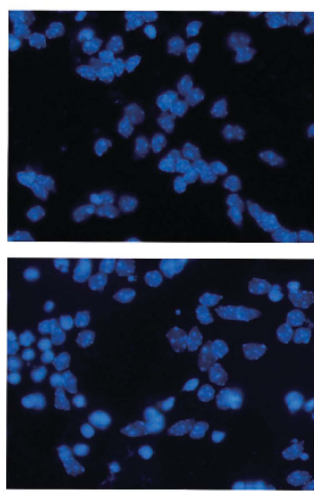

Merge
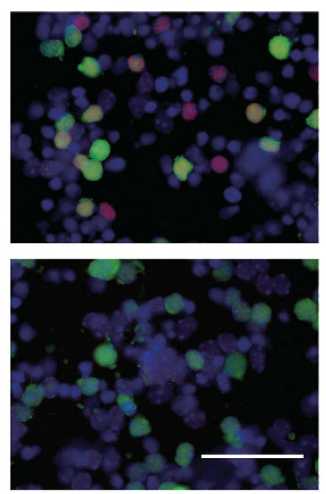

Merge
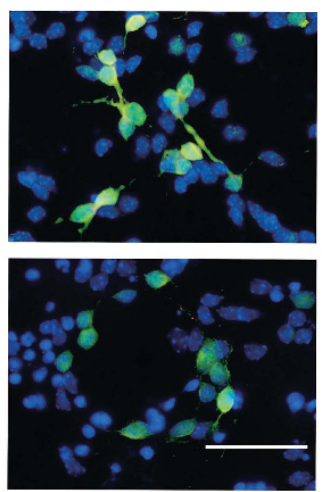

Figure 4 Knockdown of Stox1 enhances proliferation and delays the differentiation of GNPs. (a) Quantitative RT-PCR and immunobloting confirm efficient silencing of Stox1 in shStox1 adenovirus-infected GNPs. (b) Infection of GNPs isolated from Dcx-DsRed mice with shStox1 adenovirus leads to decreased generation of Dcx-DsRed- positive cells. Representative flow cytometric plots are shown. Histograms show the percentage of Dcx-dsRed-positive cells. $(n=3)$. (c). Immunofluorescent staining indicates that Stox1 knockdown represses the differentiation of NeuN-positive granule neurons from in vitro culture of GNPs. The scale bar represents $25 \mu \mathrm{m}$. (d) Stox $1 \mathrm{knockdown}$ downregulates the differentiation of Dcx-positive granule neurons from in vitro culture of GNPs. The scale bar represents $25 \mu \mathrm{m}$. (e) The mRNA levels of Math1 are upregulated, while Dcx, Tuj1, NeuN, Neurod1, Zic1 and Zic2 are downregulated in shStox1 adenovirus-infected GNPs. (f) Knockdown of Stox1 in GNPs leads to increased cell proliferation in the presence of $\mathrm{SHH}(1 \mu \mathrm{g} / \mathrm{ml})$. Cell proliferation is assessed by EdU incorporation assay. $(n=3)$. $(\mathbf{g})$ Quantitative RT-PCR analysis demonstrates enhanced expression of Ccnd1 and reduced mRNA levels of p18, p21 and p27 in shStox1 infected GNPs cultured in the presence of $1 \mu \mathrm{g} / \mathrm{ml}$ SHH. $\left({ }^{* \star *} P<0.001,{ }^{* \star} P<0.01,{ }^{*} P<0.05\right.$, data are shown as means \pm S.E.M.) 
e
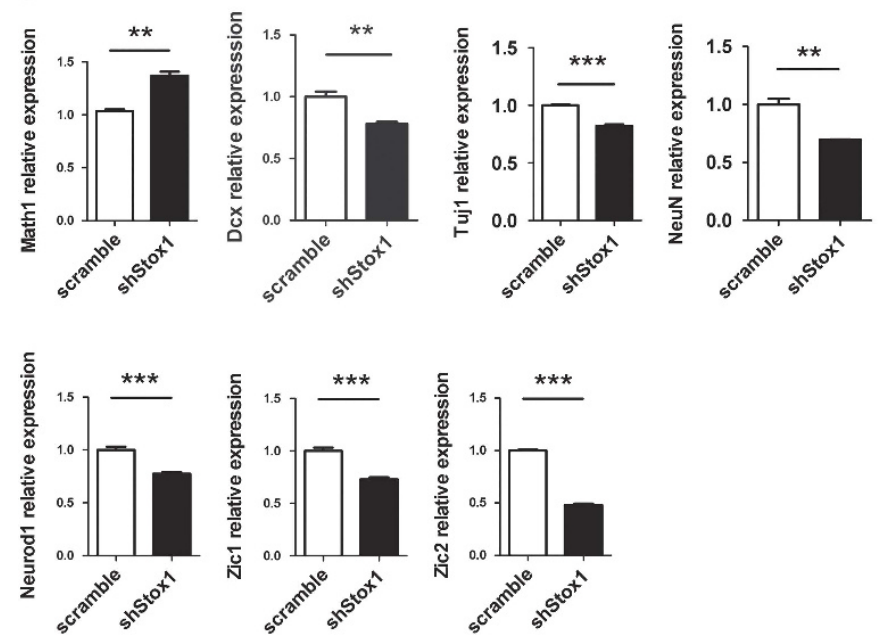

f
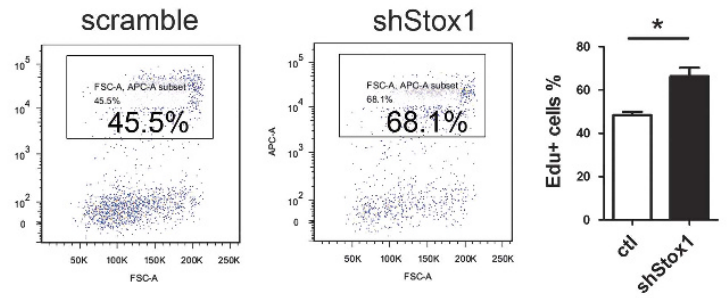

g
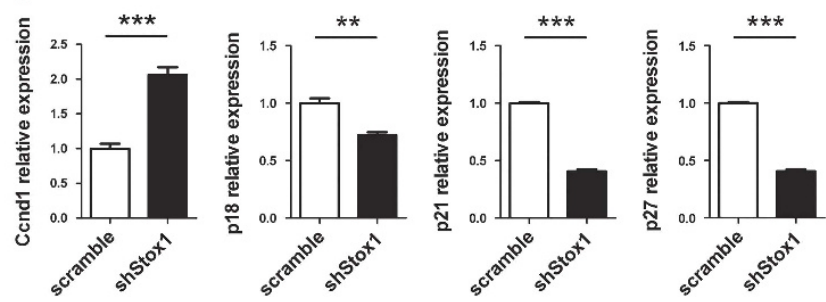

Figure 4 Continued

expression driven by enhancer $\mathrm{A}$ and enhancer $\mathrm{B}$, but not fragment $C$. It is in agreement with the previous findings that enhancer $A$ and enhancer $B$ exhibited redundant activities in governing Math1 expression. ${ }^{37}$ We further designed four pairs of primers that flanked the putative Stox1-binding sites in Math1 enhancers for chromatin immunoprecipitation (ChIP) (Figure 5c). As showed in Figure 5c, there is a marked enrichment of Stox1 in Math1 enhancer $A$ and enhancer B, suggesting a binding of Stox1 to the cisregulatory elements of Math1.

To pinpoint the DNA-binding sites of Stox1 in Math1 enhancer, we introduced mutations to the putative Stox1binding site $A 1, A 2, B 1, B 2$ in enhancer $A$ and enhancer $B$, respectively (Figure $5 d$ ). As shown in Figure $5 d$, compound mutations in $\mathrm{A} 1$ and $\mathrm{A} 2$ or mutation in $\mathrm{B} 2$ alone were able to compromise the transcriptional repression effect of Stox1, suggesting they are important for the regulation of Stox 1 on Math1 transcription. Sequence alignment among site A1, A2 and $\mathrm{B} 2$ indicated MYHAACA is the potential recognition sequence. Further gel-shifting experiment is needed to verify whether Stox 1 binds directly to the proposed DNA sequence. In addition, the transcriptional induction of Math1 gene by $\mathrm{SHH}$ stimulation can be significantly abrogated by Stox1 overexpression (Figure 5e), further supporting the notion that Stox1 acted as a transcriptional suppressor of Math1.

Stox1 overexpression inhibits the Patched $^{+/-}$tumor growth in vivo. Aberrant activation of $\mathrm{SHH}$ signaling occurs in $\sim 25 \%$ of human medulloblastomas (MBs) and accounts for the most prevalent MB subtype. ${ }^{38,39}$ Patched acts as a $\mathrm{SHH}$ receptor and inhibits $\mathrm{SHH}$ signaling by suppressing $\mathrm{SMO}{ }^{11}$ Mutation of Patched leads to constitutive activation of the $\mathrm{SHH}$ pathway, deregulated GNP proliferation, blockage of GNP differentiation and spontaneous MB formation in Patched+/- mice. ${ }^{11}$ We demonstrated above that Stox1 functioned as a positive regulator of GNP differentiation. We then examined whether it was involved in MB development. As shown in Figures $6 a$ and $b$, the expression of Stox1 was significantly reduced in the MB tissues from Patched $^{+/-}$mice compared with wild-type cerebellar tissues assessed by quantitative PCR and immunoblotting. To further test if the downregulation of Stox 1 in MB of Patched ${ }^{+/-}$mice was due to $\mathrm{SHH}$ signaling, $\mathrm{SHH}$ signal pathway inhibitor cyclopamine was used to treat dissociated Patched ${ }^{+/-}$MB cells. As shown in Figure 6c, cyclopamine treatment led to enhanced expression of Stox1, associated with downregulation of Math1 and Ccnd1. We next explored the role of Stox1 in $\mathrm{MB}$ by taking an overexpressing approach. Infection of Patched $^{+/}$MB cells with adenovirus expressing Stox1 resulted in marked suppression of in vivo growth of tumor cells (Figure 6d). Gene expression analysis indicated that overexpression of Stox1 in MB cells led to significant suppression of GNP cell marker Math1 and upregulation of differentiated neuron marker Tuj1 and NeuN (Figure 6e), similar to what we observed in normal cerebellum development. Taken together, those data suggested that Stox1 functioned as a tumor suppressor in $\mathrm{SHH}$ subtype of $\mathrm{MB}$ possibly by suppressing the transcriptional of Math1 and promoting GNP differentiation.

\section{Discussion}

We show in this study that Stox1 acts as a pivotal factor in cerebellar development and MB oncogenesis. In both of the two biological processes, Stox1 appears to exert its function through the same molecular mechanism, that is, through facilitating GNP differentiation by direct suppression of Math1 transcription. We demonstrate here that Stox1 binds to the Math1 enhancer and functions as a novel transcriptional suppressor of Math1. We further show that Stox1 is repressed by $\mathrm{SHH}$ activation and downregulated in Patched $^{+/-} \mathrm{MB}$ tissues. It has been reported that the increase of Math1 expression in $\mathrm{SHH}$-activated subtype of $\mathrm{MB}$ is essential for the blockage of neuronal differentiation and initiation of MB. ${ }^{19,40}$ Our findings point to Stox 1 as a crucial missing link between $\mathrm{SHH}$ signaling and Math1. Those data highlight a SHH-Stox1Math1 regulatory axis in normal cerebellar development and MB formation. 
a

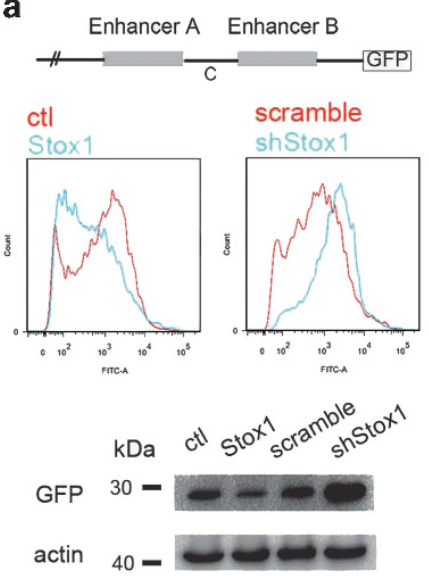

C

Enhancer A: 1-561bp

Putative Stox 1 binding sites

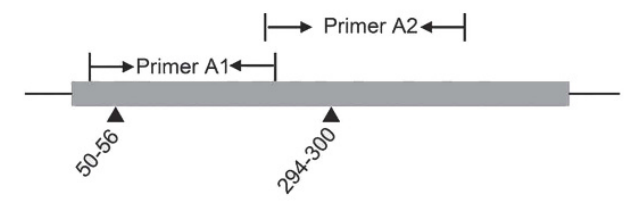

Enhancer B: 974-1385bp

Putative Stox 1 binding sites
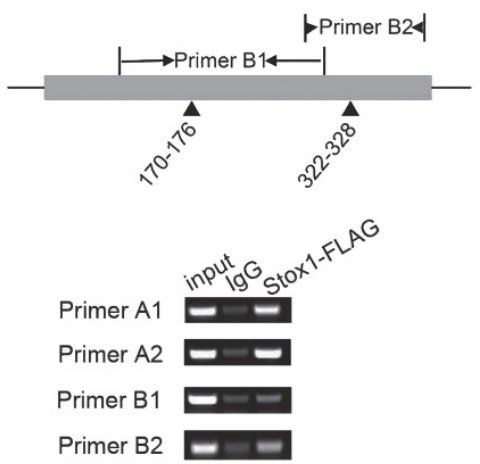
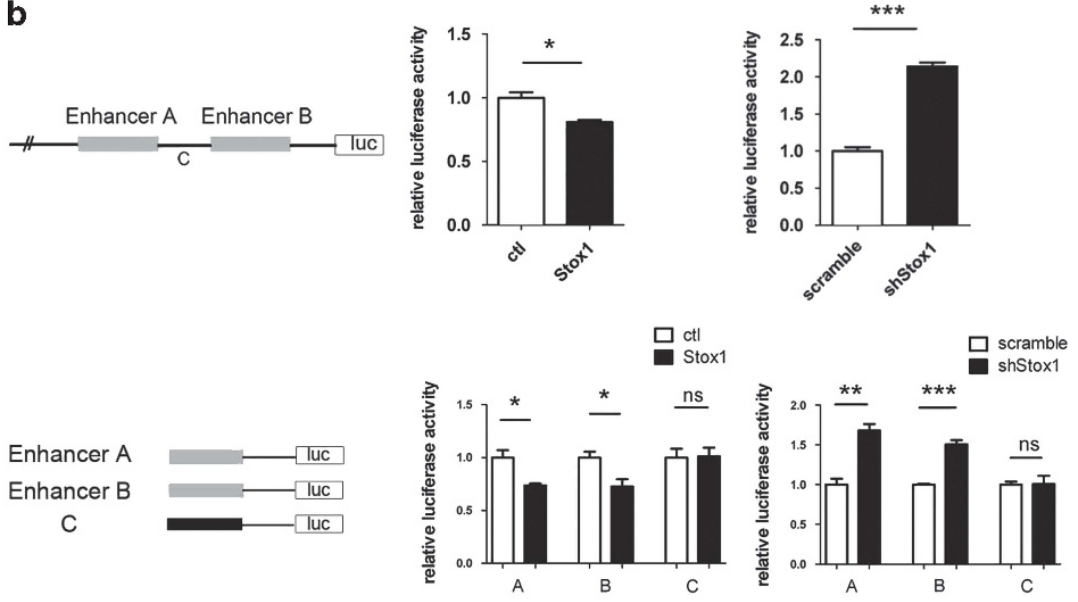

d

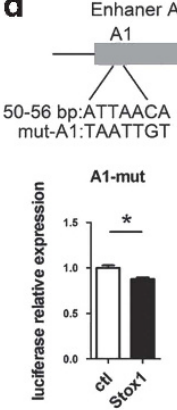

$\mathrm{A}^{2}$
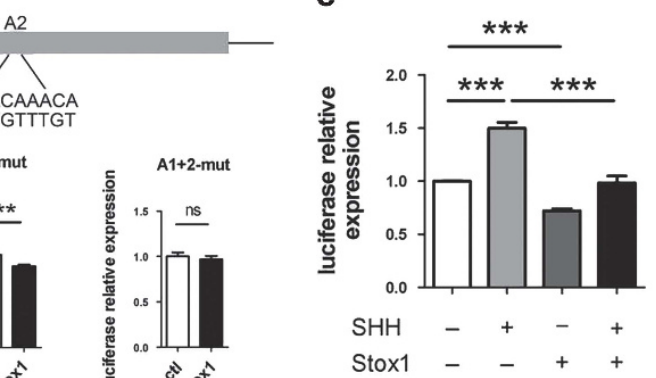

Figure 5 Stox1 acts as a transcriptional suppressor for Math1. (a) Stox1 suppresses Math1 enhancer activity. Construct with Math1 regulatory elements fusion to GFP reporter was transfected into 293T cells. Stox1 overexpression leads to GFP downregulation, whereas Stox1 knockdown by shRNA results in GFP upregulation. Representative flow cytometric plots of GFP fluorescent signal are shown in the upper panel. Immunoblotings of the GFP expression levels are shown in the lower panel. Actin was used as loading control. (b) Activities of enhancer A and B of Math1 are modulated by Stox1. The Math1 regulatory elements enhancer A, enhancer B and fragment C were fused to luciferase reporter together or separately. The different luciferase reporter constructs were transfected with the CMV-Renilla plasmid as internal control into 293T cells. Stox1 overexpression results in reduced luciferase activity in enhancer $A / B$ with fragment $C$, enhancer $A$ or enhancer $B$ plasmid transfectants, but not fragment $C$ plasmid transfectent. Stox1 knockdown displays opposite effect. (c) Semi-quantitative analysis of Stox1 binding to the enhancer of Math1 gene by the CHIP assay. Primer pairs A1, A2, B1 and B2 flanking potential Stox1-binding sites are designed in Math1 enhancer A or enhancer B. Flag tag fused Stox1-expressing plasmid were transfected into N2A cells. Anti-flag antibody was used for the immunoprecipitation. (d) The putative Stox1-binding sites A1, A2, B1 and B2 were mutated in luciferase reporter constructs. Mutated constructs were transfected with the CMV-Renilla plasmid into 293T cells. The compound mutations in A1 and A2 or mutation in B2 alone compromises the repression of luciferase reporter by Stox1 overexpression. ${ }^{* * *} P<0.001,{ }^{* *} P<0.01,{ }^{*} P<0.05$, data are shown as means \pm S.E.M.). (e) SHH stimulation leads to increased Math1 enhancer activity, which can be significantly compromised by Stox1 overexpression. Construct with Math1 enhancer fusion to luciferase reporter was transfected together with CMV-Renilla plasmid. Cells were treated with $1 \mu \mathrm{g} / \mathrm{ml} \mathrm{SHH}$ and infected with Stox1-expressing adenovirus in indicated experimental group. Luciferase activity was assessed at $48 \mathrm{~h}$ later. $\left({ }^{\star \star \star} P<0.001,{ }^{*} P<0.05\right.$, data are shown as means \pm S.E.M.)

Stox 1 is a winged helix transcription factor that structurally and functionally related to the Forkhead protein family. Several potential targets of Stox1 have been reported. CNTNAP2, a member of the neurexin family, is a negatively regulated target gene by Stox 1 in Alzheimer's disease. ${ }^{41}$ In addition, SFRS7 was found to be a target gene positively regulated by Stox 1 in glial cells. ${ }^{23}$ However, the core DNA-binding sequence has not been elucidated yet. We identify in the current study that Stox1 binds to the Math1 enhancer, probably at the MYHAACA recognition sequence similar to other forkhead family proteins. 


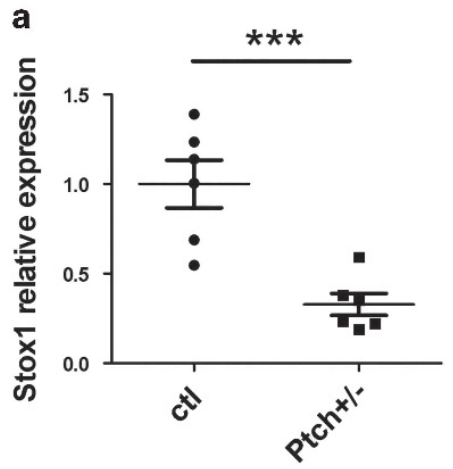

b

Stox1

$\begin{array}{lllllll}\mathrm{kDa} & \mathrm{N} & \mathrm{T} & \mathrm{N} & \mathrm{T} & \mathrm{N} & \mathrm{T}\end{array}$

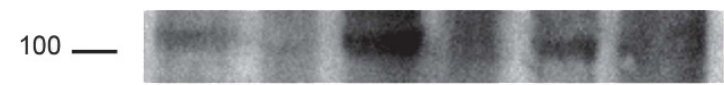

actin c

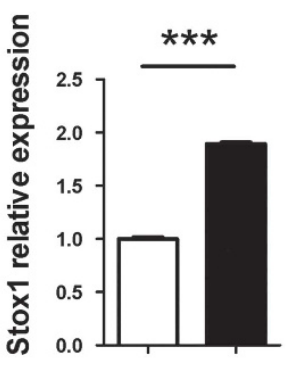

cyclopamine - +

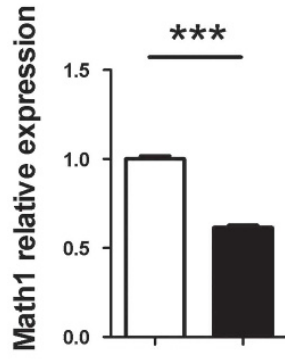

cyclopamine - $\quad+$

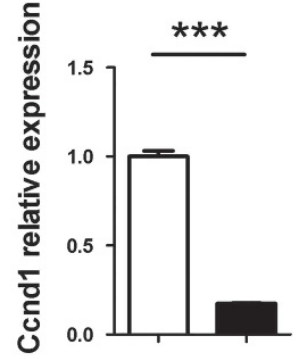

cyclopamine - +
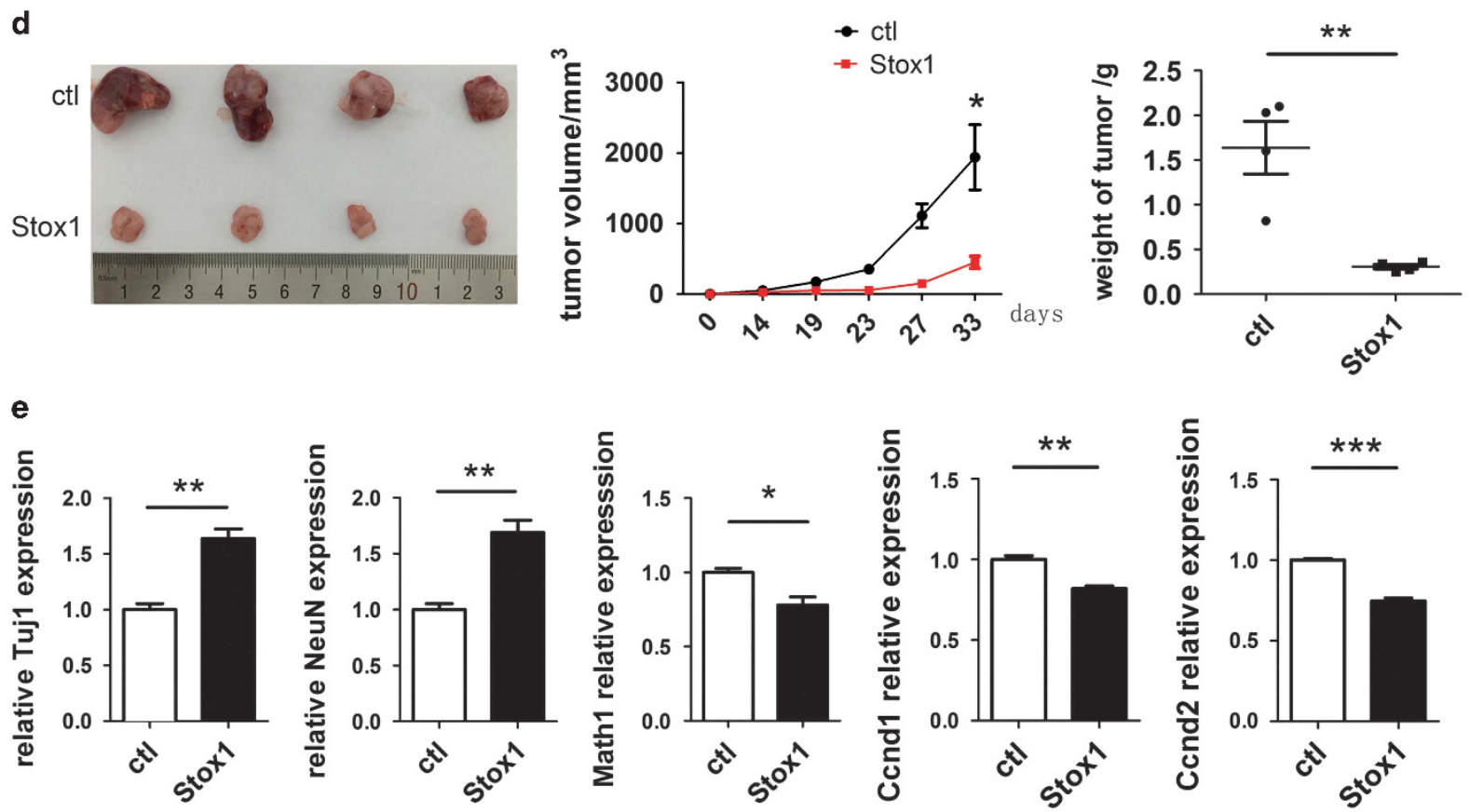

Figure 6 Stox 1 inhibits the tumor growth of SHH MB. (a) The mRNA level of Stox1 is significantly decreased in Patched ${ }^{+/-}$mouse MB samples compared with cerebellar tissues from wild-type mice. (b) The Stox1 protein amount in Patched ${ }^{+/-}$mouse MB samples is significantly lower than wild-type controls. (c) Cyclopamine treatment of Patched $^{+/-}$mouse MB cells results in enhanced transcription of Stox1 and repressed expression of Ccnd1 and Math1. (d) Stox1 overexpression significantly suppresses the xenograft growth of Patched ${ }^{+/}$MB cells $(n=4)$. (e) Increased mRNA levels of Tuj1 and NeuN and decreased mRNA levels of Math1, Ccnd1 and Ccnd2 are detected in Patched $^{+/-}$MB cells infected with Stox1-expressing adenovirus. $\left({ }^{* \star *} P<0.001,{ }^{* *} P<0.01,{ }^{\star} P<0.05\right.$, data are shown as means \pm S.E.M.)

Gel shift experiments using purified Stox1 protein will be needed to prove the direct binding of Stox 1 with the proposed DNA sites.

In view of the suppressing effects of Stox 1 on expression of Math1, it is important to point out that Math1, a bHLH transcription factor, is an important cell fate determinant not only during cerebellar development, but also during intestinal, inner ear and mechanosensary neuronal development. ${ }^{29,42-45}$ Although Math1 appears to bind to the E-box of the DNA promoter regions of specific genes by a competition 
mechanism with Notch signaling molecules ${ }^{46,47}$ or upregulates other transcription factor genes, ${ }^{19,37,48,49}$ its upstream element is poorly known. The present study shows clearly that Stox1 as an upstream suppressor of Math1 in the cerebellum. It will be interesting to determine whether Stox1 also has a role in other tissues to modulate Math1 expression. In this regard, we demonstrated in a recent paper that Stox1 promotes proliferation of inner ear epithelial cells though elevated Akt signaling pathway. ${ }^{50}$ It is also reported that Stox 1 acts as a transcriptional activator of cyclin B1 and enhances mitotic entry of glioma cells. ${ }^{51}$ Interestingly, in the current study, our data indicated that Stox1 is a negative regulator of neuronal progenitor proliferation in the cerebellum. Ectopic expression of Stox1 leads to GNP differentiation and MB inhibition. Transcriptional factors often work in complex. The distinct roles of Stox1 in different tissues are presumably owing to the presence of other co-factor or epigenetic modifiers in each specific cellular context. Previous study showed that Neurod1, Zic1 and Hic1 are possible transcriptional suppressor of Math1 in the cerebellum. ${ }^{52-54}$ It remains to be determined whether those known factors interact with Stox 1 in the regulation of Math1 transcription.

$\mathrm{SHH}$-activated $\mathrm{MB}$ constitutes the most frequently detected molecular subtype of $\mathrm{MB}$, therefore making $\mathrm{SHH}$ signaling related molecules the most promising therapeutic targets. However, drug resistance and disease relapse due to SMO mutations remain to be a major challenge for the application of current $\mathrm{SHH}$ receptor antagonists. ${ }^{14,15}$ Stox1, by its capacity to suppress the expression of Math1, the key effector of $\mathrm{SHH}$ signaling, may represent an attractive new target in $\mathrm{SHH}-$ activated subtype of MBs.

\section{Methods and Materials}

Mouse models. All experimental mice were housed in the pathogen-free animal center at Renji Hospital, Shanghai Jiaotong University with controlled temperature and humidity. Math1-GFP transgenic mice were a gift from Dr. Jane Johnson at University of Texas Southwestern Medical Center at Dallas, Dallas, TX, USA. Dcx-dsRed (009655) and Patched ${ }^{+/-}$(003081) mice were purchased from the Jackson Laboratory (Bar Harbor, ME, USA). All animal experimental protocols were approved by the Animal Research Ethics Committee of Renji Hospital.

Isolation and culture of GNPs. GNPs were isolated from postnatal day 3 or day 6 mouse cerebellum according to the procedures described before. ${ }^{3,34}$ In brief, the dissected cerebella were digested with $2 \mathrm{mg} / \mathrm{ml}$ papain (Sigma, St. Louis, MO, USA) and $0.1 \%$ DNase I (Roche, Indianapolis, IN, USA) for $15 \mathrm{~min}$, then filtered with a $70 \mu \mathrm{m}$ cell stainer (Millipore, Billerica, MA, USA). The suspension of single cells was gently placed on the $35 \% / 60 \%$ percoll gradient and was subjected to centrifugation for $10 \mathrm{~min}$ at $1800 \times \mathrm{g}$. The GNPs were collected at the interface between 35 and 60\% percoll. Dissociated GNPs were maintained in Neurobasal medium (Gibco, Waltham, MA, USA) containing B27 supplement (Gibco), 1 mM glutamine (Sigma), $100 \mathrm{U} / \mathrm{ml}$ penicillin/streptomycin, 0.45\% D-glucose and $25 \mathrm{mM}$ $\mathrm{KCL}$ in $1 \mathrm{mg} / \mathrm{ml}$ poly-d-lysine (Sigma) coated plates. For adenovirus infection, GNPs were incubated with adenovirus for $4 \mathrm{~h}$ and replaced with fresh culture medium.

Xenograft of Patched ${ }^{+/-}$MB cells. MB tissues from Patched ${ }^{+/-}$mice were harvested and then digested in $1 \mathrm{mg} / \mathrm{ml}$ collagenase (Sigma) and $0.1 \%$ DNase (Roche) for $30 \mathrm{~min}$ at $37^{\circ} \mathrm{C}$ for single cell isolation. To evaluate the impact of Stox1 on Patched ${ }^{+/-}$MB growth in vivo, dissociated Patched ${ }^{+/}$MB cells $\left(5 \times 10^{6}\right.$ cells) were infected with adenoviruses for $4 \mathrm{~h}$, then mixed with matrigel (BD Bioscience, Bedford, MA, USA) at a 1:1 ratio and injected subcutaneously into flanks of 6-week-old nude mice (SLAC, Shanghai, China). Tumor size was measured and recorded every week after inoculation. Tumor volume was calculated with the formula, Volume $=1 / 2\left(\right.$ length $\times$ width $\left.^{2}\right)$.
RNA isolation and quantitative RT-PCR. Total RNA was extracted using TRIzol reagent according to the manufacture's instruction. The CDNA syntheses were reverse transcribed from total RNA with PrimeScript RT reagent (Takara, Dalian, China). SYBR green Real-time PCR super mix (Toyobo, Osaka, Japan) was used for quantitative PCR. Quantitative PCR was performed on the Step one Plus RT-PCR Systems (Applied Biosystems, Waltham, MA, USA). The primers for quantitative PCR were provided in the following table.

\begin{tabular}{lll} 
Gene & Forward & Reverse \\
\hline Mouse Tuj1 & TAGACCCCAGCGGCAACTAT & GTTCCAGGTTCCAAGTCCACC \\
Mouse NeuN & GGCAAATGTCGGGCAATCG & TCAATTTCCGTCCCTCTACGAT \\
Mouse Ccnd1 & GCGTACCCTGACACCAATCTC & CTCCTCTTCGCACTTCTGCTC \\
Mouse Ccnd2 & GAGTGGGAACTGGTAGTTTG & CGCACAGAGCGATGAAGGT \\
Mouse p18 & CCTTGGGGGAAGAGTTG & AAATTGGGATAGCACCTCTGAG \\
Mouse p21 & CCTGGTGATGTCCGACCTG & CCATGAGCGCATCGCAATC \\
Mouse p27 & TCAAACGTGAGAGTGTCTAACG & CCGGGCCGAAGAGATTCTG \\
Mouse Math1 & GAGTGGGCTGAGGTAAAAGAGT & GGTCGGTGTATCCAGGAG \\
Mouse actin & GGCTGTATTCCCTCCATCG & CCAGTTGGTAACAATGCCATGT \\
Mouse STOX1 & TTACCCAGGTATTGCTGTTCCC & TGAGCACCCTTCGTTTGACA \\
Mouse Neurod1 & ATGACCAATCATACAGCGAGAG & TCTGCCTCGTGTCCTCGT \\
Mouse Dcx & GGCCAAGAAGGTACGTTCTAC & AGCAACGCATCAAAACTACGAA \\
Human Stox1 & TCCAGTGCAAATGAATCCAA & CAGCGTTCCCAGAGTGGTAT \\
DsRed & GGACGGCTCCTTCATCTACA & GCTCCACGATGGTGTAGTCC
\end{tabular}

Immunoblotting. Cells or tissue samples were lysed in RIPA (Pierce, Waltham, MA, USA) supplemented with the protease inhibitor cocktail (Thermo scientific, Waltham, MA, USA). The protein concentration was assessed by a BCA assay (Thermo Scientific). Protein samples were separated by SDS-PAGE gels and then transferred to nitrocellulose membranes. Membranes were blocked with $5 \%$ fat-free milk in TBS for $1 \mathrm{~h}$, incubated with primary antibody overnight at $4{ }^{\circ} \mathrm{C}$, washed three times in TBS containing 1\% Tween20, then incubated with horseradish peroxidase conjugated secondary antibody for additional $1 \mathrm{~h}$, and developed using the SuperSignal West Pico Chemiluminescent Substrate (Pierce). Antibodies against Stox1 were purchased from Santa Cruz (sc-133266, Dallas, TX, USA). Anti- $\beta$-actin (Cell Signaling Technology, 4970, Danvers, MA, USA) was used for internal loading control.

Luciferase assays. Math1 enhancer luciferase constructs were co-transfected with CMV-Renilla plasmid (Promega, Madison, WI, USA) using lipofectamine 3000 transfection reagents (Roche). Forty-eight hours after transfection, cells were lysed and analyzed with the Dual-Luciferase Reporter Assay System (Promega). Luciferase activity values were normalized to the Renilla value.

ChIP assay. The N2A cells were transfected with pETP-flag-Stox1 plasmid. Forty-eight hours after transfection, cells were harvested and fixed with $1 \%$ formaldehyde. ChIP assay was performed using EZ-ChIP Kit from millipore according to the manufacturer's instruction. Antibody against FLAG (Sigma) was used to immunoprecipitate the Stox1-DNA complex. Semi-quantitative PCR was conducted to assess the enrichment of Stox 1 in indicated Math1 cis-regulatory elements. The primers for PCR amplification were provided in the following table.

\begin{tabular}{lll} 
& Forward & Reverse \\
\hline Math1-A1 & GTTTGGCAGCTCCCTCTCTCA & TTGGATCTGACTTTGGAAGGTAG \\
Math1-A2 & TACCTTCCAAAGTCAGATCCAA & AAAAGTGTGGTGAGCCAAAAC \\
Math1-B1 & GAGAGAAGTCGGAGGAAGATAA & GACGACGGTGTTAAATGGG \\
Math1-B2 & TTAACACCGTCGTCACCCTC & AGAGGCTCTGGCTTCTGTAAACT
\end{tabular}

Immunofluorescent staining. The GNPs were cultured on the poly-D-lysine coated glass cover slides. For immunofluorescent stainings, the slides were first fixed in $4 \%$ paraformaldehyde for $10 \mathrm{~min}$, then treated with $0.5 \%$ Triton X-100 for $15 \mathrm{~min}$ and blocked with $10 \%$ donkey serum for $1 \mathrm{~h}$ at room temperature. Indicated primary antibody was then applied to the overnight at $4{ }^{\circ} \mathrm{C}$. After washing with PBS for three times, slides were incubated with secondary antibody for $1 \mathrm{~h}$ at room temperature. After extensive washing with PBS, slides were mounted with mounting medium containing DAPI (Life Technologies, Waltham, MA, USA). Antibodies used in the immunofluorescent staining experiments were rabbit anti-Stox $1(1: 200$, Santa Cruze, sc-133266), mouse anti-NeuN (1:200, Millipore, MAB337), mouse anti-Tuj1 (1: 200, Cell Signaling Technologies, 4466), goat anti-Dcx (1:200, Santa Cruze, sc-8066) chicken anti-GFP $(1: 1000$, Abcam, ab13970), rabbit anti-RFP ( 1 : 200, Abcam, ab62341, Cambridge, MA, USA).

Edu assay. GNPs for Edu assay were cultured in suspension with $\mathrm{SHH}$ $(1 \mu \mathrm{g} / \mathrm{ml})$ to retain the proliferation ability. After $4 \mathrm{~h}$ of adenovirus infection, the 
culture medium were changed to $\mathrm{SHH}$ containing medium. After $24 \mathrm{~h}$, the cells were treated with EdU at the concentration of $10 \mu \mathrm{M}$ for $12 \mathrm{~h}$. The EdU staining was performed using the Click-iT EdU Alexa Fluor 647 Imaging Kit (Invitrogen, Waltham, MA, USA) according to the manufacturer's instructions. The samples were analyzed with a BD Aria flow cytometer. Flow cytometric data were analyzed by the FlowJo software.

Plasmid constructs. The Math1 enhancer GFP plasmid was kindly shared by Dr. Jane Johnson. ${ }^{37,55}$ The full-length or truncated Math1 enhancer were amplified by PCR from the Math1 enhancer GFP plasmid, and then cloned to a minimal promoter driven luciferase reporter pGL3.G basic, respectively. The mutations of enhancer $\mathrm{A} 1, \mathrm{~A} 2, \mathrm{~B} 1$ and $\mathrm{B} 2$ were performed by PCR directly mutating the target sequence on enhancer $A$, enhancer $B$ as showed in Figure 3 . The primers for luciferase constructs cloning were provided in the following table. For the adenoviruses of Stox1 overexpression and knockdown, the CDNA or shRNA sequence of Stox 1 was cloned to the adenoviral vector pAVsi 1.1. The sequence of shStox 1 is GCTGTGCTATAGCTGATAT.

\begin{tabular}{|c|c|c|}
\hline & Forward & Reverse \\
\hline $\begin{array}{l}\text { enhancer A } \\
\text { enhancer B } \\
\text { enhancer-C } \\
\text { mut-A1 } \\
\text { mut-A2 } \\
\text { mut-B1 } \\
\text { mut-B2 }\end{array}$ & $\begin{array}{l}\text { GAAGATCTGGTCGACAGATCTCAATGAAG } \\
\text { GAAGATCTTTGCTTCTGGCTCCTAACTG } \\
\text { GAAGACTGCACATCTACAGAAAGGGA } \\
\text { CACCCCTAATTGTAGCTGTAACATATAGCTGCA } \\
\text { ACCCAACGAGTACAAGAGTCAGCACTTCTAAAG } \\
\text { AGCGGCTCTGTATAGAGGGGCTGGCAGAGGC } \\
\text { GGCCTCGAGTTGTCGGCCTCCTCCTCGTAG }\end{array}$ & 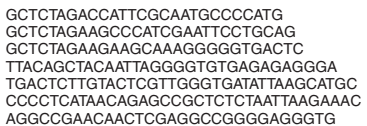 \\
\hline
\end{tabular}

Statistical analysis. Data are presented as mean \pm S.E.M. Statistical analyses were performed using Student's $t$-test.

\section{Conflict of Interest}

The authors declare no conflict of interest.

Acknowledgements. We thank Dr Jane Johnson for the generous sharing of MATH1 enhancer GFP plasmid. The study is supported by funds to W-Q Gao from the Chinese Ministry of Science and Technology (2012CB966800 and 2013CB945600), the National Natural Science Foundation of China (NSFC, 81130038 and 81372189), the Shanghai Health Bureau Key Discipline and Specialty Foundation, the Shanghai Education Committee Key Discipline and Specialty Foundation (J50208) and the KC Wong foundation, and by funds to HH Zhu from the NSFC (81270627), the State Key Laboratory of Oncogenes and Related Genes (90-1603), and Shanghai Institutions of Higher Learning (The Program for Professor of Special Appointment (Young Eastern Scholar)), and by funds to Chenlu Zhang from doctoral Innovation Fund Projects from Shanghai Jiao Tong University School of Medicine.

1. Roussel MF, Hatten ME. Cerebellum development and medulloblastoma. Curr Top Dev Biol 2011; 94: 235-282.

2. Zhang C, Guo Y, Cui J, Zhu HH, Gao WQ. Cytokeratin 18 is not required for morphogenesis of developing prostates but contributes to adult prostate regeneration. BioMed Res Int 2013; 2013: 576472

3. Gao WO, Heintz N, Hatten ME. Cerebellar granule cell neurogenesis is regulated by cell-cell interactions in vitro. Neuron 1991; 6: 705-715.

4. Gao WQ, Hatten ME. Neuronal differentiation rescued by implantation of Weaver granule cell precursors into wild-type cerebellar cortex. Science 1993; 260: 367-369.

5. Espinosa JS, Luo L. Timing neurogenesis and differentiation: insights from quantitative clonal analyses of cerebellar granule cells. J Neurosci 2008; 28: 2301-2312.

6. Yang R, Wang M, Wang J, Huang X, Yang R, Gao WQ. Cell division mode change mediates the regulation of cerebellar granule neurogenesis controlled by the sonic hedgehog signaling. Stem Cell Rep 2015; 5: 816-828.

7. Edmondson JC, Hatten ME. Glial-guided granule neuron migration in vitro: a high-resolution time-lapse video microscopic study. J Neuroscience 1987; 7: 1928-1934.

8. Northcott PA, Korshunov A, Witt H, Hielscher T, Eberhart CG, Mack S et al. Medulloblastoma comprises four distinct molecular variants. J Clin Oncol 2011; 29: 1408-1414.

9. Kool M, Koster J, Bunt J, Hasselt NE, Lakeman A, van Sluis P et al. Integrated genomics identifies five medulloblastoma subtypes with distinct genetic profiles, pathway signatures and clinicopathological features. PloS One 2008; 3: e3088.

10. Thompson MC, Fuller C, Hogg TL, Dalton J, Finkelstein D, Lau CC et al. Genomics identifies medulloblastoma subgroups that are enriched for specific genetic alterations. J Clin Oncol 2006; 24: 1924-1931.

11. Goodrich LV, Milenkovic L, Higgins KM, Scott MP. Altered neural cell fates and medulloblastoma in mouse patched mutants. Science 1997; 277: 1109-1113.
12. Schuller U, Heine VM, Mao J, Kho AT, Dillon AK, Han YG et al. Acquisition of granule neuron precursor identity is a critical determinant of progenitor cell competence to form Shh-induced medulloblastoma. Cancer Cell 2008; 14: 123-134.

13. Yang ZJ, Ellis T, Markant SL, Read TA, Kessler JD, Bourboulas M et al. Medulloblastoma can be initiated by deletion of Patched in lineage-restricted progenitors or stem cells. Cancer Cell 2008; 14: 135-145.

14. Rudin CM, Hann CL, Laterra J, Yauch RL, Callahan CA, Fu L et al. Treatment of medulloblastoma with hedgehog pathway inhibitor GDC-0449. N Engl J Med 2009; 361: 1173-1178.

15. Ng JM, Curran T. The Hedgehog's tale: developing strategies for targeting cancer. Nat Rev Cancer 2011; 11: 493-501.

16. Akazawa C, Ishibashi M, Shimizu C, Nakanishi S, Kageyama R. A mammalian helix-loophelix factor structurally related to the product of Drosophila proneural gene atonal is a positive transcriptional regulator expressed in the developing nervous system. J Biol Chem 1995; 270: 8730-8738.

17. Ben-Arie N, McCall AE, Berkman S, Eichele G, Bellen HJ, Zoghbi HY. Evolutionary conservation of sequence and expression of the bHLH protein Atonal suggests a conserved role in neurogenesis. Hum Mol Genet 1996; 5: 1207-1216.

18. Zhao H, Ayrault O, Zindy F, Kim JH, Roussel MF. Post-transcriptional down-regulation of Atoh1/Math1 by bone morphogenic proteins suppresses medulloblastoma development. Genes Dev 2008; 22: 722-727.

19. Flora A, Klisch TJ, Schuster G, Zoghbi HY. Deletion of Atoh1 disrupts Sonic Hedgehog signaling in the developing cerebellum and prevents medulloblastoma. Science 2009; 326: 1424-1427.

20. Klisch TJ, Xi Y, Flora A, Wang L, Li W, Zoghbi HY. In vivo Atoh1 targetome reveals how a proneural transcription factor regulates cerebellar development. Proc Natl Acad Sci USA 2011; 108: 3288-3293.

21. Aragaki M, Tsuchiya K, Okamoto R, Yoshioka S, Nakamura T, Sakamoto N et al. Proteasomal degradation of Atoh1 by aberrant Wnt signaling maintains the undifferentiated state of colon cancer. Biochem Biophys Res Commun 2008; 368: 923-929.

22. van Dijk M, Mulders J, Poutsma A, Konst AA, Lachmeijer AM, Dekker GA et al. Maternal segregation of the Dutch preeclampsia locus at 10q22 with a new member of the winged helix gene family. Nat Genet 2005; 37: 514-519.

23. van Abel D, Holzel DR, Jain S, Lun FM, Zheng YW, Chen EZ et al. SFRS7-mediated splicing of tau exon 10 is directly regulated by STOX1A in glial cells. PloS One 2011; 6: e21994.

24. Feng G, Yi P, Yang Y, Chai Y, Tian D, Zhu Z et al. Developmental stage-dependent transcriptional regulatory pathways control neuroblast lineage progression. Development 2013; 140: 3838-3847.

25. Kee Y, Hwang BJ, Sternberg PW, Bronner-Fraser M. Evolutionary conservation of cell migration genes: from nematode neurons to vertebrate neural crest. Genes Dev 2007; 21: 391-396.

26. Hatten ME. Neuronal regulation of astroglial morphology and proliferation in vitro. J Cell Biol 1985; 100: 384-396.

27. Rao MS, Shetty AK. Efficacy of doublecortin as a marker to analyse the absolute number and dendritic growth of newly generated neurons in the adult dentate gyrus. Eur $\mathrm{J}$ Neurosci 2004; 19: 234-246.

28. Wechsler-Reya RJ, Scott MP. Control of neuronal precursor proliferation in the cerebellum by Sonic Hedgehog. Neuron 1999; 22: 103-114.

29. Ben-Arie N, Bellen HJ, Armstrong DL, McCall AE, Gordadze PR, Guo Q et al. Math1 is essential for genesis of cerebellar granule neurons. Nature 1997; 390: 169-172.

30. Lumpkin EA, Collisson T, Parab P, Omer-Abdalla A, Haeberle H, Chen P et al. Math1-driven GFP expression in the developing nervous system of transgenic mice. Gene Expr Patterns 2003; 3: 389-395.

31. Francis F, Koulakoff A, Boucher D, Chafey P, Schaar B, Vinet MC et al. Doublecortin is a developmentally regulated, microtubule-associated protein expressed in migrating and differentiating neurons. Neuron 1999; 23: 247-256.

32. Gleeson JG, Lin PT, Flanagan LA, Walsh CA. Doublecortin is a microtubule-associated protein and is expressed widely by migrating neurons. Neuron 1999; 23: 257-271.

33. Wang X, Qiu R, Tsark W, Lu Q. Rapid promoter analysis in developing mouse brain and genetic labeling of young neurons by doublecortin-DsRed-express. J Neurosci Res 2007; 85: 3567-3573.

34. Lee HY, Greene LA, Mason CA, Manzini MC. Isolation and culture of post-natal mouse cerebellar granule neuron progenitor cells and neurons. J Vis Exp 2009. doi: 10.3791/990.

35. Overdier DG, Porcella A, Costa RH. The DNA-binding specificity of the hepatocyte nuclear factor 3/forkhead domain is influenced by amino-acid residues adjacent to the recognition helix. Mol Cell Biol 1994; 14: 2755-2766.

36. Carlsson P, Mahlapuu M. Forkhead transcription factors: key players in development and metabolism. Dev Biol 2002; 250: 1-23.

37. Helms AW, Abney AL, Ben-Arie N, Zoghbi HY, Johnson JE. Autoregulation and multiple enhancers control Math1 expression in the developing nervous system. Development 2000; 127: 1185-1196.

38. Gilbertson RJ, Ellison DW. The origins of medulloblastoma subtypes. Annu Rev Pathol 2008; 3: $341-365$.

39. Gibson P, Tong Y, Robinson G, Thompson MC, Currle DS, Eden C et al. Subtypes of medulloblastoma have distinct developmental origins. Nature 2010; 468: 1095-1099.

40. Ayrault O, Zhao H, Zindy F, Qu C, Sherr CJ, Roussel MF. Atoh1 inhibits neuronal differentiation and collaborates with Gli1 to generate medulloblastoma-initiating cells. Cancer Res 2010; 70: 5618-5627. 
41. van Abel $D$, Michel $O$, Veerhuis $R$, Jacobs $M$, van Dijk $M$, Oudejans $C B$. Direct downregulation of CNTNAP2 by STOX1A is associated with Alzheimer's disease. $J$ Alzheimer Dis 2012; 31: 793-800.

42. Yang Q, Bermingham NA, Finegold MJ, Zoghbi HY. Requirement of Math1 for secretory cell lineage commitment in the mouse intestine. Science 2001; 294: 2155-2158.

43. Shroyer NF, Helmrath MA, Wang VY, Antalffy B, Henning SJ, Zoghbi HY. Intestine-specific ablation of mouse atonal homolog 1 (Math1) reveals a role in cellular homeostasis. Gastroenterology 2007; 132: 2478-2488.

44. Bermingham NA, Hassan BA, Price SD, Vollrath MA, Ben-Arie N, Eatock RA et al. Math1: an essential gene for the generation of inner ear hair cells. Science 1999; 284: 1837-1841.

45. Ben-Arie N, Hassan BA, Bermingham NA, Malicki DM, Armstrong D, Matzuk M et al. Functional conservation of atonal and Math1 in the CNS and PNS. Development 2000; 127: 1039-1048.

46. Gazit R, Krizhanovsky V, Ben-Arie N. Math1 controls cerebellar granule cell differentiation by regulating multiple components of the Notch signaling pathway. Development 2004; 131: 903-913.

47. Powell LM, Zur Lage PI, Prentice DR, Senthinathan B, Jarman AP. The proneural proteins Atonal and Scute regulate neural target genes through different E-box binding sites. Mol Cell Biol 2004; 24: 9517-9526.

48. Saba R, Johnson JE, Saito T. Commissural neuron identity is specified by a homeodomain protein, Mbh1, that is directly downstream of Math1. Development 2005; 132: 2147-2155.
49. Kawauchi D, Saito T. Transcriptional cascade from Math1 to Mbh1 and Mbh2 is required for cerebellar granule cell differentiation. Dev Biol 2008; 322: 345-354.

50. Nie X, Zhang K, Wang L, Ou G, Zhu H, Gao WQ. Transcription factor STOX1 regulates proliferation of inner ear epithelial cells via the AKT pathway. Cell Prolif 2015; 48 : 209-220

51. Abel D, Abdul-Hamid O, Dijk M, Oudejans CB. Transcription factor STOX1A promotes mitotic entry by binding to the CCNB1 promotor. PloS One 2012; 7: e29769.

52. Pan N, Jahan I, Lee JE, Fritzsch B. Defects in the cerebella of conditional Neurod1 null mice correlate with effective $\mathrm{Tg}($ Atoh1-cre) recombination and granule cell requirements for Neurod1 for differentiation. Cell Tissue Res 2009; 337: 407-428.

53. Ebert PJ, Timmer JR, Nakada Y, Helms AW, Parab PB, Liu Y et al. Zic1 represses Math1 expression via interactions with the Math1 enhancer and modulation of Math1 autoregulation. Development 2003; 130: 1949-1959.

54. Briggs KJ, Corcoran-Schwartz IM, Zhang W, Harcke T, Devereux WL, Baylin SB et al. Cooperation between the Hic1 and Ptch1 tumor suppressors in medulloblastoma. Genes Dev 2008; 22: 770-785.

55. Lumpkin EA, Collisson T, Parab P, Omer-Abdalla A, Haeberle H, Chen P et al. Math1-driven GFP expression in the developing nervous system of transgenic mice. Gene Expr Patterns 2003; 3: 389-395. 